(W)

Check for

updates

Cite as

Nano-Micro Lett.

(2021) 13:131

Received: 16 March 2021

Accepted: 4 May 2021

Published online: 29 May 2021

(C) The Author(s) 2021

\section{A General Self-Sacrifice Template Strategy to 3D Heteroatom-Doped Macroporous Carbon for High-Performance Potassium-Ion Hybrid Capacitors}

\author{
Junwei $\mathrm{Li}^{1}$, Xiang $\mathrm{Hu}^{1}{ }^{凶}$, Guobao Zhong ${ }^{1}$, Yangjie Liu ${ }^{1}$, Yaxin $\mathrm{Ji}^{1}$, Junxiang Chen ${ }^{1}$,
} Zhenhai Wen ${ }^{1}$

\title{
HIGHLIGHTS
}

- A newly versatile self-sacrifice method to fabricate selenium and nitrogen co-doped 3D macroporous carbon was developed.

- The $\mathrm{K}^{+}$storage mechanism of Se and $\mathrm{N}$ active sites was deeply studied by systematic electrochemical characterization combined with theoretical calculations.

- The assembled K-ion hybrid capacitor possesses high energy/power densities and excellent cycling stability for practical applications.

ABSTRACT Potassium-ion hybrid capacitors (PIHCs) tactfully combining capacitor-type cathode with battery-type anode have recently attracted increasing attentions due to their advantages of decent energy density, high power density, and low cost; the mismatches of capacity and kinetics between capacitor-type cathode and battery-type anode in PIHCs yet hinder their overall performance output. Herein, based on prediction of density functional theory calculations, we find $\mathrm{Se} / \mathrm{N}$ co-doped porous carbon is a promising candidate for $\mathrm{K}^{+}$storage and thus develop a simple and universal self-sacrifice template method to fabricate Se and N co-doped three-dimensional (3D) macroporous carbon (Se/N-3DMpC), which features favorable properties of connective hierarchical pores, expanded interlayer structure, and rich activity site for boosting pseudocapacitive activity and kinetics toward $\mathrm{K}^{+}$storage anode and enhancing capacitance performance for the reversible anion adsorption/desorption cathode. As expected, the as-assembled PIHCs full cell with a working voltage as

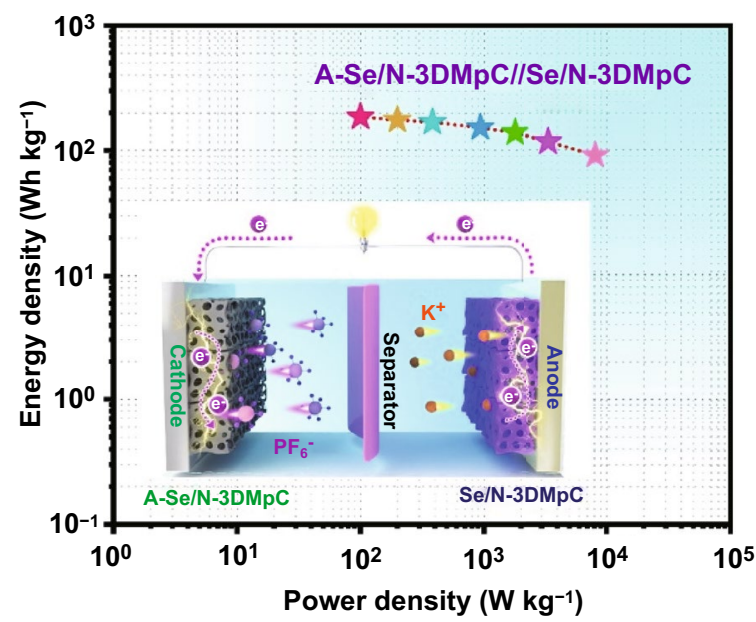
high as 4.0 V delivers a high energy density of $186 \mathrm{Wh} \mathrm{kg}^{-1}$ and a power output of $8100 \mathrm{~W} \mathrm{~kg}^{-1}$ as well as excellent long service life. The proofof-concept PIHCs with excellent performance open a new avenue for the development and application of high-performance hybrid capacitors.

KEYWORDS Potassium-ion hybrid capacitors; Self-sacrifice template; Se/N co-doped; 3D macroporous; Long service life

Xiang Hu, huxiang@ fjirsm.ac.cn; Zhenhai Wen,wen@fjirsm.ac.cn

1 CAS Key Laboratory of Design and Assembly of Functional Nanostructures, and Fujian Provincial Key Laboratory of Nanomaterials, Fujian Institute of Research On the Structure of Matter, Chinese Academy of Sciences, Fuzhou, Fujian 350002, People's Republic of China 


\section{Introduction}

Potassium-ion hybrid capacitor (PIHC), as an emerging new energy storage device, has recently been proposed to bridge the gap between high power density of potassiumion batteries (PIBs) and high energy density of supercapacitor (SC) by integrating complementary merits of the two systems [1-4]. Unfortunately, the potassium-ion storage anode usually suffers from the issues of dramatic volume variation and fast capacity fading because of the intrinsic large-sized $\mathrm{K}^{+}$, which causes slow redox reaction kinetics behavior upon $\mathrm{K}^{+}$intercalation/deintercalation process [5-8]. In addition, the activated carbon, as the typical capacitor-type cathode of PIHCs, shows fast charge/discharge rate, while it tends to deliver limited capacitance $[6,9]$. Therefore, the development of desired electrode materials to overcome the imbalance of kinetics and capacity between the anode and cathode in PIHCs is urgently needed, yet remains grand challenge so far [10-12].

A diversity of materials, such as metal alloys [13, 14], selenides [5], oxides [15, 16], MXene [17, 18], and carbon-based material [19] have been studied as battery-type anodes. Among them, carbon-based materials [10, 20-23] with the intercalation mechanism are considered as one of the most promising anode candidates for large-scale practical applications thanks to the attractive merits of low cost and high electrical conductivity. Therefore, various carbon-based materials, including graphene [24], hard carbon microspheres [25], soft carbon [26], hard-soft composite carbon [27], nanocrystalline graphite [28], and carbon nanofibers [29], have been extensively studied as anode of PIHCs. Recently, the theoretical calculations show that the $\mathrm{N}$-doped, P/N co-doped, and S/N co-doped carbon-based materials can effectively adjust the localized electrons near the heteroatoms, increase electron negativity of defective sites, and improve the electrical conductivity of carbon, thereby promoting the potassium storage performance [3, $4,30]$. Nevertheless, there remains space to further improve the overall performances of PIHCs for stepping forward its practical application. On the one hand, relatively narrow interlayer space in the traditional battery-type carbon anode likely led to low capacity and unsatisfactory rate. On the other hand, activated carbon (AC) is commonly employed as cathode material for PIHCs due to its large specific surface area (SSA) [9], and its rather low level of activity sites limited the specific capacity of cathode, presenting another critical factor encumbering the overall performance of PIHCs.

Herein, density functional theory (DFT) calculations are performed to screen selenium (Se) and nitrogen (N) codoped carbon as one promising material for $\mathrm{K}^{+}$storage. To this end, we develop a newly versatile self-sacrifice method to fabricate Se and N co-doped three-dimensional (3D) macroporous carbon (Se/N-3DMpC) by using $\mathrm{SeO}_{2}$ powder as the Se source and self-sacrifice template. The desired features in Se/N-3DMpC, including connective hierarchical pores, expanded interlayer structure, good conductivity, and rich activity site, are greatly beneficial to facilitate $\mathrm{K}^{+}$reversible insertion/extraction and to increase the redox sites for capacitor-type cathode. As expected, the resultant $\mathrm{Se} / \mathrm{N}-3 \mathrm{DMpC}$ exhibits superior $\mathrm{K}^{+}$storage capability and impressively high capacitance and thus shows promise to be applied as both anode and cathode of PIHCs by manifesting high energy/power density and long cycling stability.

\section{Experimental Section}

\subsection{Synthesis of Se/N-3DMpC}

$100 \mathrm{mg}$ chitosan, $200 \mathrm{mg}$ urea, and $200 \mathrm{mg} \mathrm{SeO}{ }_{2}$ were first ground to mix well. The mixture was then transferred into an autoclave and heated to $200{ }^{\circ} \mathrm{C}$ for $3 \mathrm{~h}$ to gain the precursor. The precursor was then loaded in the porcelain boat and placed at the middle position of a quartz tube. The Se/N$3 \mathrm{DMpC}$ was ultimately obtained through high-temperature treatment at $800{ }^{\circ} \mathrm{C}$ for $2 \mathrm{~h}$ under Ar atmosphere with a heating rate of $5{ }^{\circ} \mathrm{C} \mathrm{min}^{-1}$.

\subsection{Synthesis of Se-3DMpC, N-pC, and Pure-C}

The procedure for the preparation of $\mathrm{Se}-3 \mathrm{DMpC}$ is similar to $\mathrm{Se} / \mathrm{N}-3 \mathrm{DMpC}$ except that the urea was removed. The procedure for the preparation of $\mathrm{N}$-doped porous carbon $(\mathrm{N}-\mathrm{pC})$ is similar to $\mathrm{Se} / \mathrm{N}-3 \mathrm{DMpC}$ except that the $\mathrm{SeO}_{2}$ was removed. The procedure for the preparation of pure carbon (pure-C) is similar to $\mathrm{Se} / \mathrm{N}-3 \mathrm{DMpC}$ except that the $\mathrm{SeO}_{2}$ and urea were removed. 


\subsection{Synthesis of A-Se/N-3DMpC}

As-prepared Se/N-3DMpC and $\mathrm{KOH}$ were thoroughly mixed with the mass ratio of 1:4. The mixture was heated at $800{ }^{\circ} \mathrm{C}$ for $1 \mathrm{~h}$ under $\mathrm{Ar}$ atmosphere with a heating rate of $5{ }^{\circ} \mathrm{C} \mathrm{min}^{-1}$. The activated sample was washed with $1.2 \mathrm{M}$ $\mathrm{HCl}$ aqueous solution to remove additional $\mathrm{KOH}$ and then washed with deionized water (DI) for several times. Finally, the A-Se/N-3DMpC was freeze-dried for more than $72 \mathrm{~h}$ at $-40{ }^{\circ} \mathrm{C}$ with a freeze-dryer under 0.05 mbar of pressure.

\subsection{Synthesis of PVP-, Starch-, and Glucose-Based Se-Doped 3DMpC and Se/N Co-Doped 3DMpC}

The procedure for the preparation of PVP-, starch-, and glucose-based Se-doped $3 \mathrm{DMpC}$ is similar to $\mathrm{Se}-3 \mathrm{DMpC}$ except that the chitosan was replaced by PVP, starch, and glucose. The procedure for the preparation of PVP-, starch-, and glucose-based $\mathrm{Se} / \mathrm{N}$ co-doped $3 \mathrm{DMpC}$ is similar to $\mathrm{Se} / \mathrm{N}-3 \mathrm{DMpC}$ except that the chitosan was replaced by PVP, starch, and glucose.

\subsection{Material Characterizations}

The morphologies of synthesized samples were inspected by field emission scanning electron microscopy (FESEM, Hitachi SU-8020). Field emission transmission electron microscopy and high-resolution transmission electron microscopy (FETEM, Tecnai F20) were used to observe the detailed structures of as-prepared samples. The X-ray diffraction (XRD, Miniflex 600 powder X-ray diffractometer with $\mathrm{Cu} \mathrm{K} \alpha$ radiation at a scan rate of $5^{\circ} \mathrm{min}^{-1}$ ) and Raman spectrum (LabRam HR800) and X-ray photoelectron spectroscopy (XPS, ESCALAB 250Xi, Thermo Fisher) were employed to analyze the defects and detailed structures. Nitrogen adsorption-desorption isotherms were analyzed by an intelligent gravimetric sorption analyser (IGA100B). The pore-size distribution was achieved employing density functional theory (DFT) model. The Se/N-3DMpC electrodes were taken out of the half-battery at different stages in an argon-filled glove box and then were washed with diethyl carbonate before ex situ Raman, XPS, XRD, SEM, and TEM tests.

\subsection{Electrochemical Measurements}

The Se/N-3DMpC, Se-3DMpC, and N-pC electrodes were prepared by coating a slurry containing active materials, acetylene black, and sodium carboxymethyl cellulose in a weight ratio of $8: 1: 1$ on copper foil. Then, the as-prepared electrode was dried at $100{ }^{\circ} \mathrm{C}$ overnight and compressed at $5 \mathrm{MPa}$ pressure. The mass loading of the active materials on each electrode was around $1.0-1.2 \mathrm{mg} \mathrm{cm}^{-2}$. All the electrodes were assembled with potassium metal counter electrode and Whatman glass fibers into a 2032 model cells in an Ar-filled glove box ( $<0.01 \mathrm{ppm}$ of oxygen and water). $0.8 \mathrm{M} \mathrm{KPF}_{6}$ in ethylene carbonate (EC)/diethyl carbonate $(1: 1, \mathrm{v} / \mathrm{v})$ was employed as the electrolyte. The electrochemical measurements of half-batteries were performed by a LAND CT2001 test system between 0.01 and $3.0 \mathrm{~V}$ at room temperature. The cyclic voltammetry (CV) curves $\left(0.1 \mathrm{mV} \mathrm{s}^{-1}\right)$ were obtained by a CHI660E electrochemical workstation. Electrochemical impedance spectroscopy was carried out at a frequency range from $0.01 \mathrm{~Hz}$ to $100 \mathrm{kHz}$.

A coin-type PIHC full cell was constructed employing the pre-potassiation Se/N-3DMpC anode and A-Se/N-3DMpC cathode. The mass ratio of $\mathrm{Se} / \mathrm{N}-3 \mathrm{DMpC}$ (anode) and A-Se/N-3DMpC (cathode) was 1:4. The A-Se/N-3DMpC cathode was constructed by coating a slurry containing A-Se/N-3DMpC (80wt\%), acetylene black (10 wt $\%$ ), and sodium carboxymethyl cellulose (10 $\mathrm{wt} \%$ ) on aluminum foil. Before the assembling of the PIHCs devices, the Se/N$3 \mathrm{DMpC}$ anode was activated in half batteries at $0.1 \mathrm{~A} \mathrm{~g}^{-1}$ for three cycles and ended with a potassiation state of $0.01 \mathrm{~V}$. The energy $\left(\mathrm{E}, \mathrm{Wh} \mathrm{kg}^{-1}\right)$ and power densities $\left(\mathrm{P}, \mathrm{W} \mathrm{kg}^{-1}\right)$ of assembled PIHCs were calculated employing the equations as follows:

$P=\Delta V \times i$

$E=P \times t / 3600$

$\Delta V=\left(V_{\max }+V_{\min }\right) / 2$

where $t(\mathrm{~s})$ is the discharge time, $i\left(\mathrm{~A} \mathrm{~g}^{-1}\right)$ is the charge/ discharge current, $V_{\max }(\mathrm{V})$ is the discharge potential excluding the IR drop, and $V_{\min }(\mathrm{V})$ is the potential at the end of discharge voltages. 


\subsection{Calculation Method}

The density functional theory (DFT) calculations were performed with periodic super-cells under the generalized gradient approximation (GGA) using the Perdew-Burke-Ernzerhof (PBE) function for exchange-correlation. The interaction of ion-electron is described by projected augmented wave (PAW) pseudopotentials. The Kohn-Sham orbitals were expanded in a plane-wave basis set with a kinetic energy cutoff of 30 Ry and the charge density cutoff of 300 Ry. The Fermi surface effects have been treated by the smearing technique of Methfessel and Paxton, using a smearing parameter of $0.02 \mathrm{Ry}$. The Brillouin zones were sampled with a k-point mesh of $1 \times 1 \times 1$. The calculation model of the pure, $\mathrm{Se}-, \mathrm{N}-$, and dual $(\mathrm{Se}, \mathrm{N})-$ doped carbon was constructed with $5 \times 5$ lateral periodicity shown in Fig. S1. The vacuum layer was $\sim 15 \AA$ to remove the interaction of adjacent atomic slabs in the $z$ direction. The nudged elastic band (NEB) method was used to evaluate the transition state and activation energy for one $\mathrm{K}$-ion migrate from one hollow site to the nearest hollow site in the carbon. The graphitic interlayer spacing of the pure-C, $\mathrm{N}-\mathrm{pC}, \mathrm{Se}-3 \mathrm{DMpC}$, and $\mathrm{Se} / \mathrm{N}-3 \mathrm{DMpC}$ was set at 3.4, 3.6, 3.88 , and 4.04, respectively, according to the results of XRD (Fig. 2g). By adding the adsorbate in each model and then the geometry structures were optimized again (shown in Fig. S2). Subsequently, the structure of potassium ions adsorbed on different carbon models can be obtained, and the corresponding $\Delta E_{\mathrm{a}}$ can be calculated by Eq. 4 :

$\Delta E_{\mathrm{a}}=E_{\text {total }}-E_{\text {adsorbate }}-E_{\text {structure }}$

where $E_{\text {total }}$ is the total energy of compound, $E_{\text {adsorbate }}$ is the energy of adsorbate, and $E_{\text {structure }}$ is the energy of corresponding structure. Additionally, the energy of one potassium atom is calculated by the energy of per potassium atom in potassium metal phase. Our DFT calculations are implemented by the PW and NEB modules contained in the Quantum ESPRESSO distribution [31].

\section{Results and Discussion}

\subsection{DFT Analysis of the Effect of Se and N Dopants on Potassium-Ion Storage}

To explore the role of Se and $\mathrm{N}$ co-dopants in carbonbased material for PIHCs, the DFT calculations were conducted. The adsorption energy $\left(\Delta E_{\mathrm{a}}\right)$ of $\mathrm{K}^{+}$for the diversity of geometry configurations is imported as the descriptor for adsorption stability. Therefore, the models of pure carbon, Se-doped, N-doped, and Se/N co-doped carbon were constructed as host materials with optimization (Fig. S1). The $\Delta E_{\mathrm{a}}$ of $\mathrm{K}^{+}$on the activity sites in all configurations was calculated (Fig. S2). The pure carbon shows a $\Delta E_{\mathrm{a}}$ of $0.47 \mathrm{eV}$ (Fig. S2a), suggesting the dopant-free carbon cannot offer an effective adsorption for $\mathrm{K}^{+}$. With importing $\mathrm{N}$ element, it was found that the $\mathrm{K}^{+}$adsorption on the site neighbor to the edge $\mathrm{N}$ species is obviously enhanced (Fig. S2b). Therefore, $\mathrm{N}$ doping can effectively improve the adsorption capacity for $\mathrm{K}^{+}(-1.60 \mathrm{eV})$ on carbon materials. Meanwhile, Se doping in the carbon matrix can also enhance the interaction between $\mathrm{K}^{+}$and carbon, affording a $\Delta E_{\mathrm{a}}$ of $-0.04 \mathrm{eV}$ (Fig. S2c). Additionally, the larger radius of Se atom can cause obvious protrusions in the carbon surfaces, increasing the interlayer spacing between the carbon layers and thus fortifying the capacity for potassium-ion storage (Fig. S1c). Furthermore, the $\Delta E_{\mathrm{a}}$ of $\mathrm{K}^{+}$on the Se-doped carbon can be further enhanced with the additional doping of $\mathrm{N}$, resulting in a favorable $\Delta E_{\mathrm{a}}$ of $-1.61 \mathrm{eV}$ for $\mathrm{K}^{+}$adsorption (Fig. S2d). Overall, N/Se co-doping in carbon-based materials can be a promising strategy to promote the $\mathrm{K}^{+}$ storage performance.

To further investigate the bonding properties of the adsorbed $\mathrm{K}^{+}$in each model, the charge density difference was calculated by subtracting the charge density of $\mathrm{K}^{+}$and each configuration from the corresponding compound (Fig. 1a-d). Compared with undoped carbon, single heteroatoms ( $\mathrm{Se}$ or $\mathrm{N}$ ) carbon shows higher density of transferred charges, confirming that single doping of $\mathrm{Se}$ or $\mathrm{N}$ can increase the adsorption ability of $\mathrm{K}^{+}$on the carbon surface. Moreover, the highest density of transferred charges between the adsorbed $\mathrm{K}^{+}$and substrate can be observed in the Se/N co-doped carbon, demonstrating that co-doping of $\mathrm{Se} / \mathrm{N}$ is more conducive to improving the adsorption ability of $\mathrm{K}^{+}$on the carbon surface. To obtain an insightful view of the doping effects, we analyzed the partial density of states (PDOS) of p orbitals of $\mathrm{N}$ and Se atoms in the $\mathrm{Se}-, \mathrm{N}-$, and $\mathrm{Se} / \mathrm{N}$-doped carbon to investigate the electronic structure (Fig. S3). The results show that the introduction of Se atom can effectively improve the PDOS of $\mathrm{N}$ atom near the Fermi level and vice versa, finally enhancing the adsorption energy of 

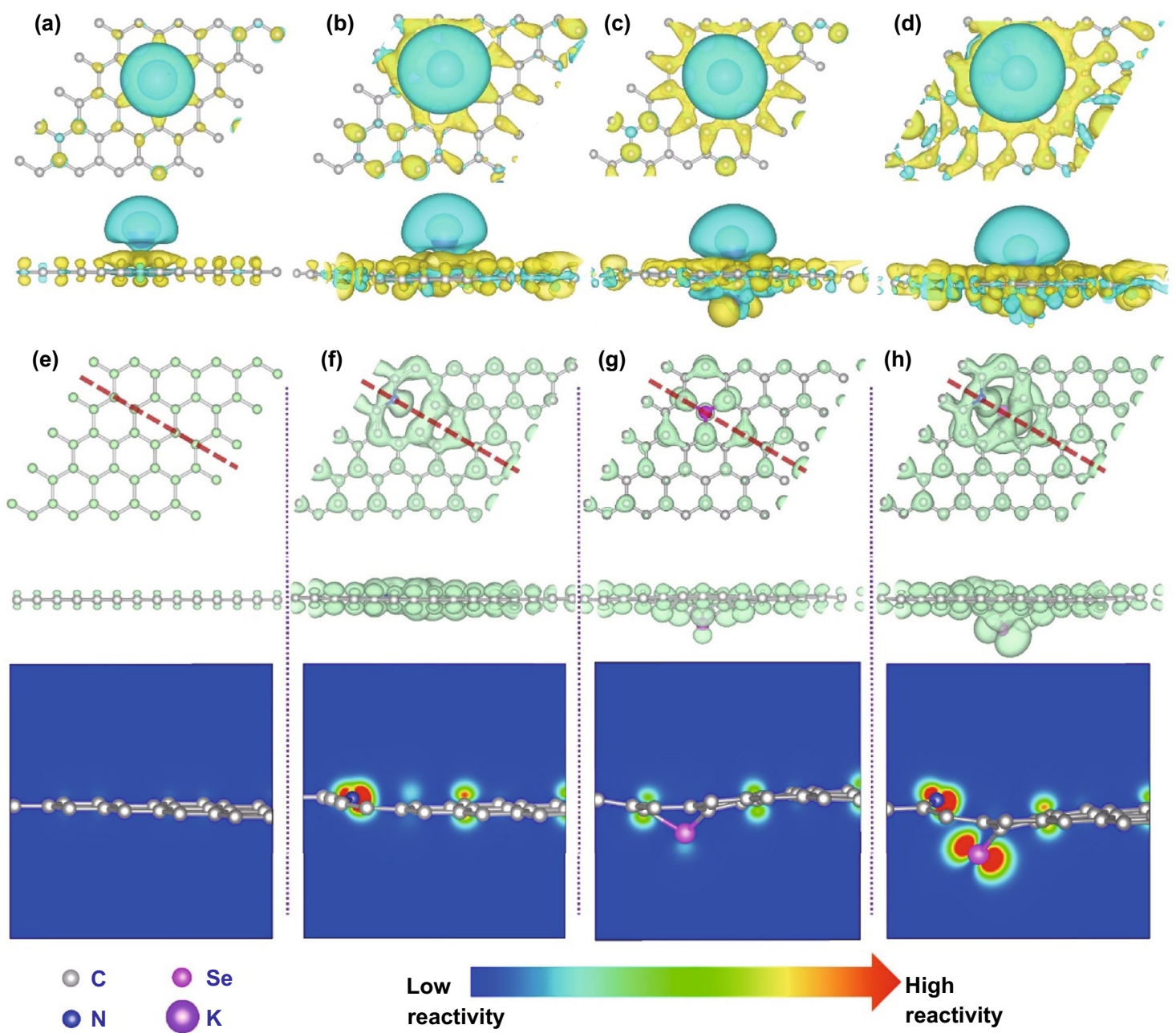

(f)

(g) $2 a_{0} a_{0}$

(h)
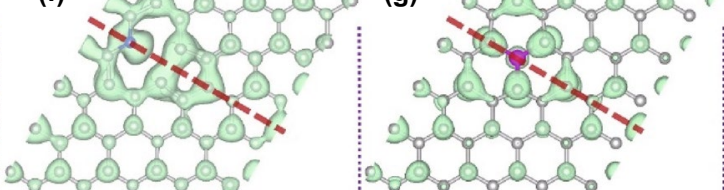
$0_{0} 0_{0} a_{0} a_{0} x^{2}$

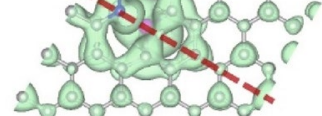
2) 0 o. $0 x$

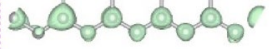

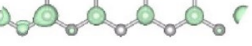
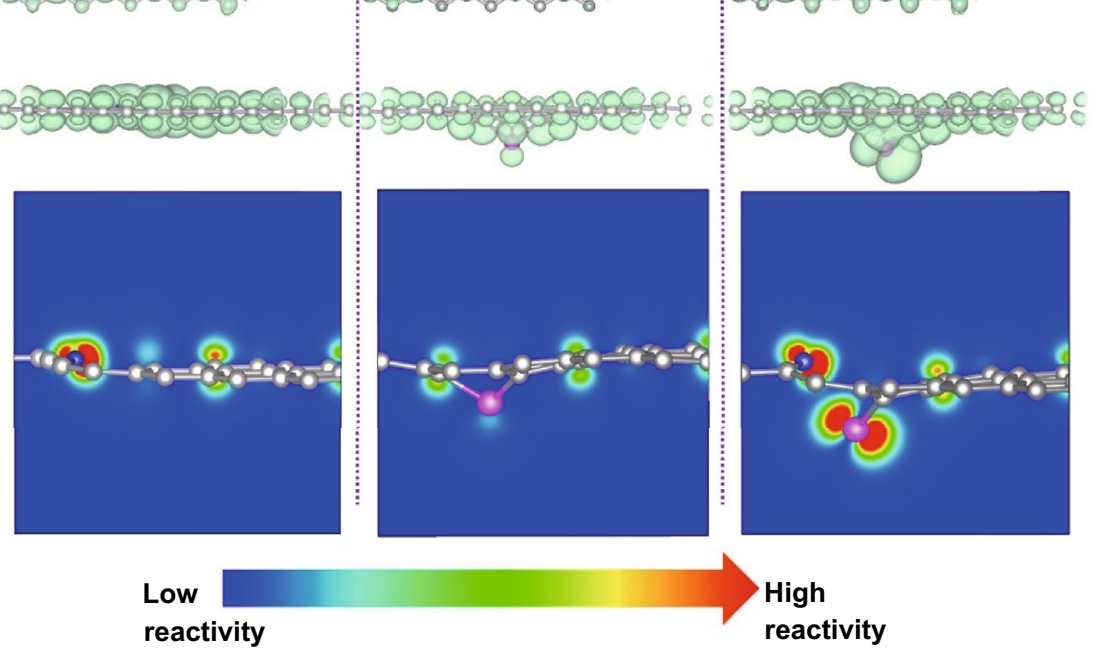

Fig. 1 Top and side illustration of the calculated charge density difference of the $\mathrm{K}^{+}$adsorbed on a pure carbon, $\mathbf{b} \mathrm{N}$-doped carbon, $\mathbf{c}$ Se-doped carbon, and $\mathbf{d} \mathrm{Se} / \mathrm{N}$ co-doped carbon. Top and side illustration of the local Fermi softness $\left[\mathrm{S}_{\mathrm{F}}(\mathrm{r})\right]$ and the corresponding sectional reactivity heat map for the active sites of the e pure carbon, $\mathbf{f} \mathrm{N}$-doped carbon, $\mathbf{g}$ Se-doped carbon, and $\mathbf{h} \mathrm{Se} / \mathrm{N}$ co-doped carbon, where the red and blue colors indicate the higher and lower reactivity, i.e., how easy it bonds with other atoms. The charge density isosurface is set to $0.004 \mathrm{e} \AA^{-3}$ in all models

$\mathrm{K}^{+}$in the Se/N co-doped carbon [32]. Furthermore, the $\mathrm{Se} / \mathrm{N}$ co-doped carbon shows higher reactivity than others according to the associated Fermi softness analysis $\left[S_{F}(r)\right]$ (Fig. 1e-h), which is consistent with the results of PDOS analysis [33]. The local $S_{F}(r)$ is the weighted sum of the contributions from the frontier orbitals, where the higher spatial proportion of the same $S_{F}(r)$ suggests the associated active site can have a stronger bond with the potassium ion. The above results clearly demonstrate that the PDOS near the corresponding Fermi level can be mutually strengthened with the co-doping of Se and N in carbon, resulting in an increased adsorption energy for
$\mathrm{K}^{+}$, and endowing the $\mathrm{Se} / \mathrm{N}$ co-doped carbon be a promising electrode material for PIHCs.

\subsection{Synthesis and Characterizations of the $\mathrm{Se} / \mathrm{N}-3 \mathrm{DMpC}$}

Steered by the theoretical calculation, we designed a simple one-pot route to synthesize 3D macroporous carbon ( $\mathrm{Se} / \mathrm{N}$ 3DMpC) with connective hierarchical pores, as illustrated in Fig. 2a. First, the mixtures of chitosan (CS), urea, and $\mathrm{SeO}_{2}$ were preheated at $200{ }^{\circ} \mathrm{C}$ for $3 \mathrm{~h}$, during which the 
(a)
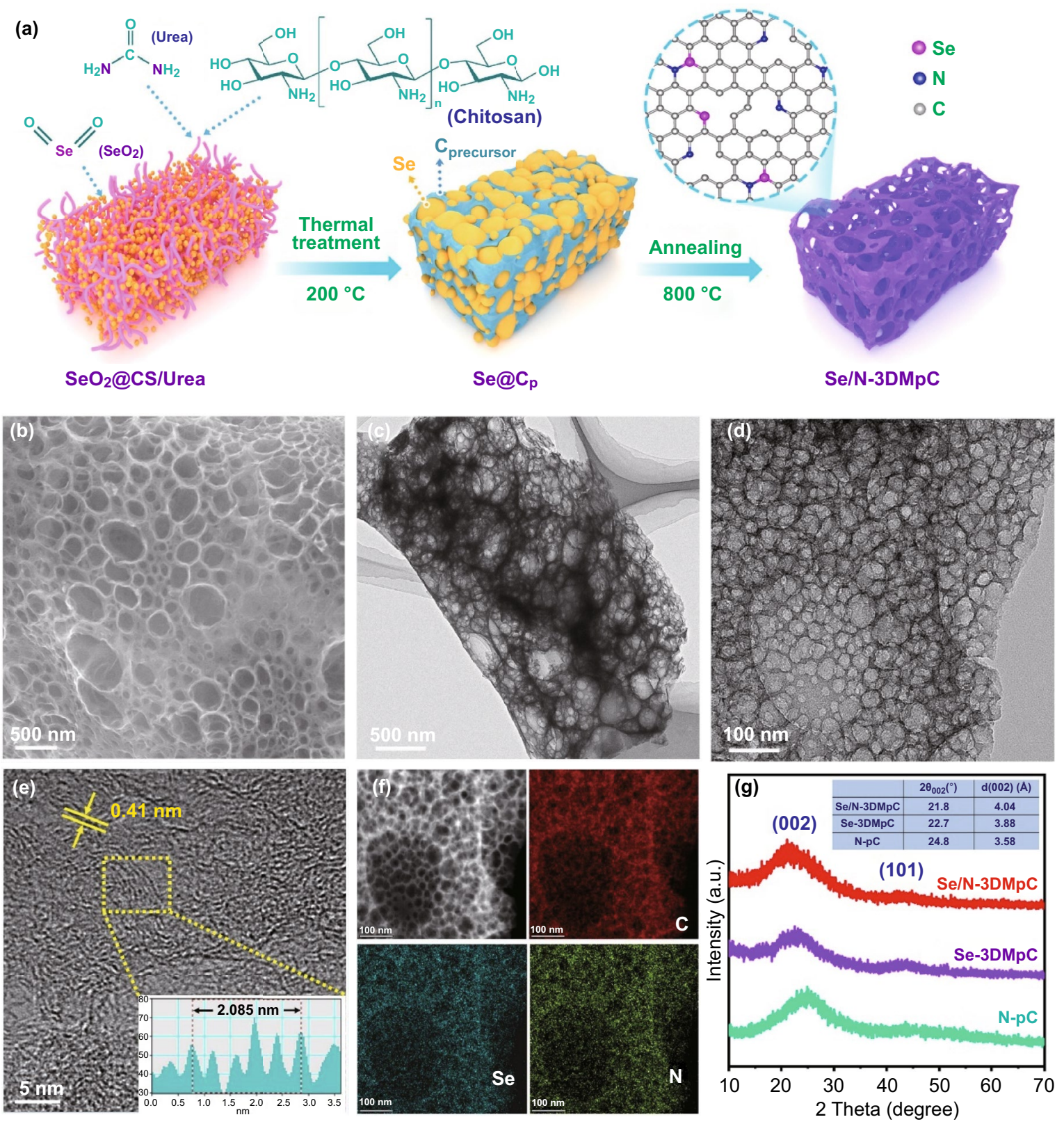

Fig. 2 a Schematic illustration for preparation of Se/N-3DMpC. b FESEM images, c, d TEM images, e HRTEM image, f HAADF-STEM image and EDS elemental mappings of the Se/N-3DMpC, $\mathbf{g}$ XRD pattern of the Se/N-3DMpC, Se-3DMpC, and N-pC

$\mathrm{SeO}_{2}$ particles tend to be encapsulated by polymer evolved from CS and urea, and then gradually reduced to Se with increasing the temperature. The subsequent pyrolysis at $800{ }^{\circ} \mathrm{C}$ under $\mathrm{Ar}$ atmosphere yields the $\mathrm{Se} / \mathrm{N}-3 \mathrm{DMpC}$ after evaporating the Se particles and carbonized the polymer. $\mathrm{SeO}_{2}$ here is not only used as the template for generating interconnective macroporous structure but also applied as the Se source for doping. For comparison, Se-doped 3D macroporous carbon (Se-3DMpC) without using urea, $\mathrm{N}$-doped microporous carbon (N-pC) without adding selenium source, and pure carbon (pure-C) without urea and selenium source were also synthesized by the similar route to prepare $\mathrm{Se} / \mathrm{N}-3 \mathrm{DMpC}$.

FESEM and TEM were employed to study the process of the formation of the Se/N-3DMpC. The sphere-like Se particles were embedded in carbon precursor matrix before 
pyrolyzation, as verified by the SEM images, the EDS element mapping, and the XRD of the samples obtained at $200{ }^{\circ} \mathrm{C}$ (Fig. S4). The obtained Se/N-3DMpC and Se$3 \mathrm{DMpC}$ obviously show macroporous structure thanks to removing Se particles upon high-temperature pyrolysis (Figs. $2 \mathrm{~b}$ and S5, S6a, b). The nitrogen adsorption-desorption tests indicate both $\mathrm{Se} / \mathrm{N}-3 \mathrm{DMpC}$ and $\mathrm{Se}-3 \mathrm{DMpC}$ consist of hierarchical porous structure with considerably high surface area of 711.86 and $652.50 \mathrm{~m}^{2} \mathrm{~g}^{-1}$, respectively (Fig. S7). Notably, the N-pC obtained without using $\mathrm{SeO}_{2}$ displays a large amount of stacking thin carbon sheets (Fig. S8), indicating that the gas produced during the high-temperature pyrolysis of urea is beneficial to the formation of thin carbon during the carbonization process. As shown in the TEM images in Figs. 2c-d and S6c-d, both Se/N-3DMpC and Se$3 \mathrm{DMpC}$ indeed have a hierarchical interconnective porous structure, which is caused by the removal of Se template and gas generated in the process of pyrolysis. High-resolution TEM (HRTEM) image reveals that the Se/N-3DMpC has an expanded interlayer distance of $0.41 \mathrm{~nm}$ (Fig. 2e), which can be conducive to the fast kinetic of insertion/extraction of $\mathrm{K}^{+}$. As expected, the interlayer spacing of $\mathrm{Se} / \mathrm{N}-3 \mathrm{DMpC}$ is significantly larger than that of Se-3DMpC $(0.39 \mathrm{~nm})$ and $\mathrm{N}-\mathrm{pC}(0.36 \mathrm{~nm})$, respectively (Figs. S6e and S8e). The high-angle annular dark-field scanning transmission electron microscopy (HAADF-STEM) image and the corresponding EDS mappings demonstrate homogeneous distribution of Se, $\mathrm{N}$, and $\mathrm{C}$ in the porous skeleton of $\mathrm{Se} / \mathrm{N}-3 \mathrm{DMpC}$ (Fig. 2f). Interestingly, by replacing different carbon sources (e.g., glucose, starch, and PVP), Se-doped and Se/N co-doped 3D macroporous carbon (3DMpC) can also be obtained (Figs. S9-S12), proving the general applicability of the preparation method.

The enlarged graphite-like interlayer space in $\mathrm{Se} / \mathrm{N}-3 \mathrm{DMpC}$ was further proved by powder XRD measurement, two broad peaks of (002) and (100) diffractions indicating the formation of graphite-like carbon for all the samples. The (002) peaks of Se/N-3DMpC, Se-3DMpC, and N-pC are located at around $21.8^{\circ}, 22.7^{\circ}$, and $24.8^{\circ}$, respectively, corresponding to interlamellar spacing of 4.04, 3.88, and $3.58 \AA$ according to the Bragg's law [34]. The enlarged interlayer space of Se/N$3 \mathrm{DMpC}$ can be attributed to the abundant heteroatoms ( $\mathrm{Se}$ and N) doped in the carbon layer, which is consistent with the HRTEM results. The Raman spectroscopy was performed to further investigate the microstructure of $\mathrm{Se} / \mathrm{N}-3 \mathrm{DMpC}$, Se3DMpC, and N-pC. As shown in Fig. S13, two characteristic peaks at $1348 \mathrm{~cm}^{-1}$ (D band) and $1580 \mathrm{~cm}^{-1}$ (G band) correspond to the breathing vibration of the hybridized carbon rings and stretching vibration of $s p^{2}$-type graphitized carbon. The intensity ratio of $\mathrm{D}$ band to $\mathrm{G}$ band $\left(I_{\mathrm{D}} / I_{\mathrm{G}}\right)$ of the Se/N-3DMpC exhibits the highest value (1.10) than those of the Se-3DMpC (1.07) and N-pC (1.04), indicating more structural defects in the Se/N-3DMpC caused by the co-doping of Se and N. XPS further reveals the elemental composition and chemical bonding state of all samples (Fig. S14). The surface elemental composition of Se/N-3DMpC consisted of N (10.48 at\%) and Se (2.62 at\%) species. The high-resolution Se $3 \mathrm{~d}$ spectrum of the $\mathrm{Se} / \mathrm{N}-3 \mathrm{DMpC}$ and $\mathrm{Se}-3 \mathrm{DMpC}$ displays two fitted peaks of $\mathrm{Se} 3 \mathrm{~d}_{5 / 2}(55.7 \mathrm{eV})$ and $\mathrm{Se} 3 \mathrm{~d}_{3 / 2}(56.6 \mathrm{eV})$ that caused by the spin-orbit coupling (Fig. S15a-b). Moreover, the binding energies of these two peaks are higher than $55.2 \mathrm{eV}$ and $56.1 \mathrm{eV}$ of pure Se, respectively, suggesting that Se atoms were successfully doped into the carbon frame to form $\mathrm{Se}-\mathrm{C}$ bonds [35]. Meanwhile, a fitting peak suggesting the formation of $\mathrm{C}-\mathrm{Se} / \mathrm{C}-\mathrm{N}$ bonds was also observed in the $\mathrm{C} 1 \mathrm{~s}$ spectrum (Fig. S15c). The species of pyridinic $(398.2 \mathrm{eV})$, graphitic $(400.7 \mathrm{eV})$, pyrrolic $(399.6 \mathrm{eV})$, and oxidized $(403.3 \mathrm{eV}) \mathrm{N}$ are present in the high-resolution $\mathrm{N} 1 \mathrm{~s}$ spectrum of $\mathrm{Se} / \mathrm{N}-3 \mathrm{DMpC}$ (Fig. S15d), implying elemental $\mathrm{N}$ has also been doped in $\mathrm{Se} / \mathrm{N}-3 \mathrm{DMpC}$. Besides, the species of $\mathrm{N}$ are not detected in the survey spectrums of pure-C and Se-3DMpC (Fig. S14), illustrating that the $\mathrm{N}$ element of $\mathrm{Se} / \mathrm{N}-3 \mathrm{DMpC}$ and $\mathrm{N}-\mathrm{pC}$ was derived from urea rather than CS. The results of XPS analysis suggest the chemically bonded Se and $\mathrm{N}$ have been successfully doped into the Se/N-3DMpC, which contribute to promoting surface capacitive capacity.

\subsection{Electrochemical Performance of Se/N-3DMpC Anode}

The $\mathrm{K}^{+}$storage performance of the as-prepared carbonbased materials was investigated in potassium-ion half-cell with metallic potassium foil as both the counter and reference electrodes. Figure 3 a displays the first three cyclic voltammetry $(\mathrm{CV})$ profiles of the $\mathrm{Se} / \mathrm{N}-3 \mathrm{DMpC}$ at a scan rate of $0.1 \mathrm{mV} \mathrm{s}^{-1}$ between 0.01 and $3.0 \mathrm{~V}$. There is an obvious cathodic peak located at around $0.52 \mathrm{~V}$ in the first cathodic scan, corresponding to the irreversible reactions of the decomposition of electrolyte and the formation of a stabilized solid electrolyte interphase (SEI) film [36]. There is a sharp cathodic peak at around $0.01 \mathrm{~V}$, which is ascribed 
to the formation of $\mathrm{K}$-intercalation compound $\left(\mathrm{KC}_{8}\right)$ by insertion of $\mathrm{K}^{+}$into the interlayer [37]. In the $\mathrm{CV}$ curves of Se-3DMpC and N-pC, similar peaks are also observed (Fig. S16a-b). During the anodic process, the peak at around $0.29 \mathrm{~V}$ is related to the extraction of $\mathrm{K}^{+}$from the interlayers of Se/N-3DMpC. An anodic peak at around $1.75 \mathrm{~V}$ can be observed in the $\mathrm{CV}$ profiles of $\mathrm{Se} / \mathrm{N}-3 \mathrm{DMpC}$ and $\mathrm{Se}-$ 3DMpC electrodes, but not in N-pC electrode, which corresponds to the reaction of potassium ions with doped selenium (Figs. 3a and S16a-b) [38]. In addition, the following $\mathrm{CV}$ curves of Se/N-3DMpC almost overlap each other, suggesting a good reversibility. Compared with the $\mathrm{CV}$ curves of Se/N-3DMpC, Se-3DMpC, and N-pC electrodes, the
$\mathrm{Se} / \mathrm{N}-3 \mathrm{DMpC}$ electrode presents the largest area, indicating that $\mathrm{Se} / \mathrm{N}-3 \mathrm{DMpC}$ has the highest potassium-ion storage capacity among all samples (Fig. S16c-d).

Figure $\mathrm{S} 17 \mathrm{a}-\mathrm{c}$ presents the galvanostatic charge-discharge (GCD) curves of all species at $0.2 \mathrm{~A} \mathrm{~g}^{-1}$. The voltage plateaus are well consistent with the redox peaks observed in the $\mathrm{CV}$ curves. The initial discharging and charging capacities of Se/N-3DMpC are 757 and $411 \mathrm{mAh} \mathrm{g}^{-1}$, corresponding to an initial Coulombic efficiency (ICE) of 54.3\%. The initial capacity loss of the Se/N-3DMpC electrode might be caused by the consumption of electrolyte and the formation of the SEI layer [39]. Compared with the ICE of Se-3DMpC $(45.7 \%)$ and $\mathrm{N}-\mathrm{pC}(30.5 \%)$, the Se/N-3DMpC shows a
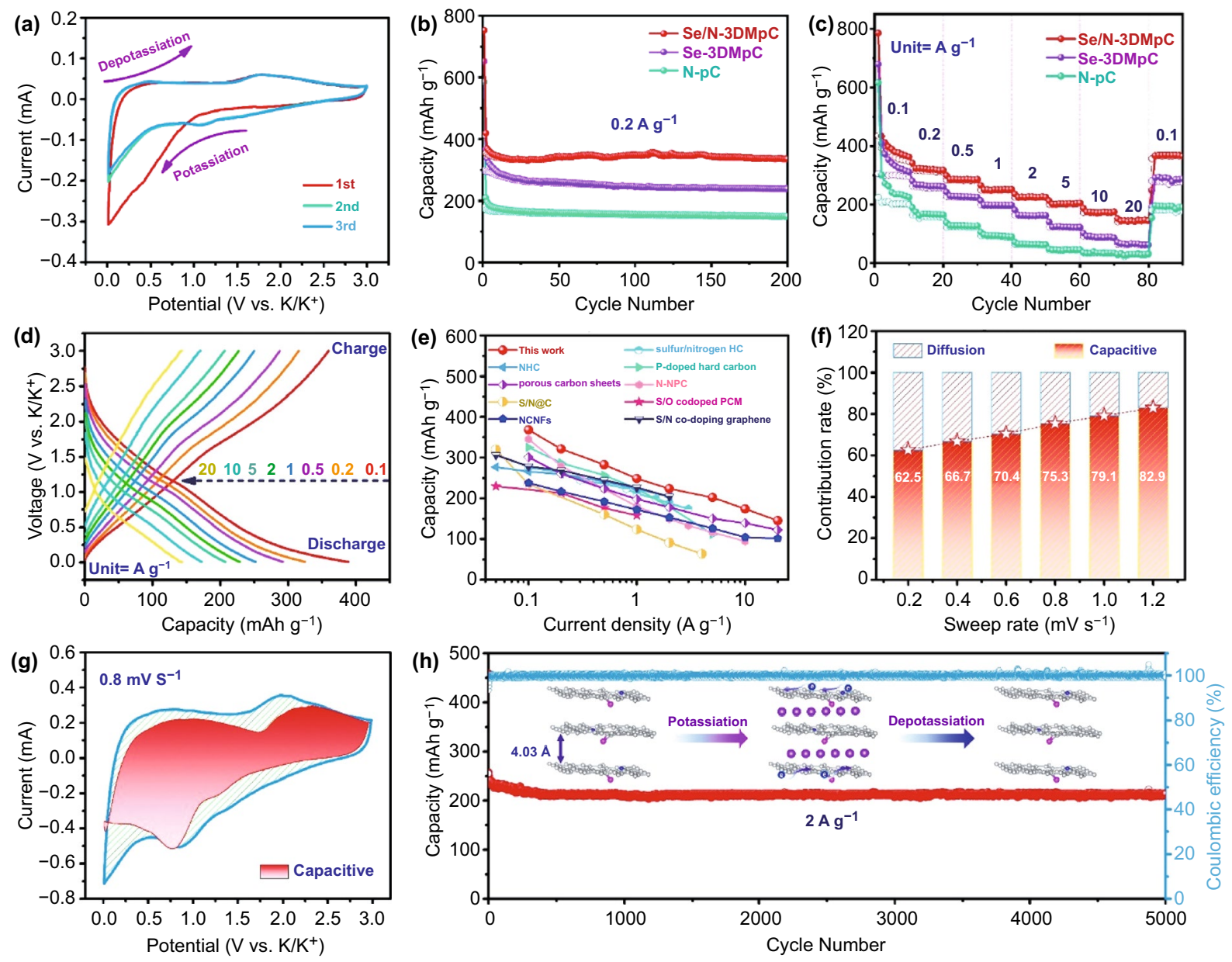

Fig. 3 a CV curves at a sweep rate of $0.1 \mathrm{mV} \mathrm{s}^{-1}$ of the Se/N-3DMpC. b Cycling performance of the Se/N-3DMpC, Se-3DMpC, and N-pC at $0.2 \mathrm{~A} \mathrm{~g}^{-1}$. c Rate performance of Se/N-3DMpC, Se-3DMpC, and N-pC. d Charge-discharge curves at diversity current densities for Se/N$3 \mathrm{DMpC}$. e Comparison of rate performance of Se/N-3DMpC with the reported carbon-based materials for PIBs. f Contribution of the surface process of the Se/N-3DMpC at different scan rates. $\mathbf{g}$ Capacitive contribution at $0.8 \mathrm{mV} \mathrm{s}^{-1}$ of Se/N-3DMpC electrode. $\mathbf{h}$ Long-term cycling performance of the $\mathrm{Se} / \mathrm{N}-3 \mathrm{DMpC}$ at a current density of $2.0 \mathrm{~A} \mathrm{~g}^{-1}$ 
considerably improved ICE (Fig. S17a-c), indicating that co-doping of Se and $\mathrm{N}$ can significantly increase the first reversible capacity of the carbon-based material. Additionally, the ICE value of the Se/N-3DMpC electrode surpasses most of the previously reported carbon-based materials (Table S1). Furthermore, the Se/N-3DMpC shows a lower voltage drop of $123 \mathrm{mV}$ than Se-3DMpC (305 mV) and $\mathrm{N}-\mathrm{HPC}(436 \mathrm{mV})$, indicating the conductivity is enhanced by co-doping with Se/N heteroatoms (Fig. S17d).

Figure $3 \mathrm{~b}$ shows the comparison of the cycling performance of the set of electrodes at $0.2 \mathrm{~A} \mathrm{~g}^{-1}$. After 200 cycles, the $\mathrm{Se} / \mathrm{N}-3 \mathrm{DMpC}$ electrode outperforms the other two electrodes by delivering a reversible specific capacity of 335 $\mathrm{mAh} \mathrm{g}^{-1}$, corresponding to a capacity retention of $95.9 \%$. Although the Se-3DMpC and N-pC electrodes exhibit a similar cyclability, their capacities after 200 cycles are only 238 and $149 \mathrm{mAh} \mathrm{g}^{-1}$, respectively. Moreover, the Coulombic efficiency of Se/N-3DMpC electrode increased to $100 \%$ after the first few cycles (Fig. S18). Figure 3c displays the rate performance of the all samples from low $\left(0.1 \mathrm{~A} \mathrm{~g}^{-1}\right)$ to ultrahigh $\left(20.0 \mathrm{~A} \mathrm{~g}^{-1}\right)$ current densities. The reversible capacities of Se/N-3DMpC are 368, 321, 283, 249, 224, 202, 174 , and $145 \mathrm{mAh} \mathrm{g}^{-1}$ at $0.1,0.2,0.5,1,2,5,10$, and $20 \mathrm{~A}$ $\mathrm{g}^{-1}$, respectively, outperforming the Se-3DMpC and N-pC counterparts, thanks to the multi-effects of 3D hierarchical porous architecture, expansion of interlayer space, and abundant heteroatoms (Se and N) doping (Fig. S19). In addition, the charge/discharge profiles of the $\mathrm{Se} / \mathrm{N}-3 \mathrm{DMpC}$ electrodes show decreased polarization relative to those of $\mathrm{Se}-3 \mathrm{DMpC}$ and N-pC (Figs. 3d and S20). Such 3D porous structural feature can reduce the volume change of carbon during $\mathrm{K}^{+}$ insertion/extraction and enhance the surface capacitancecontrolled behavior under high current density. In this context, the rate performance of $\mathrm{Se} / \mathrm{N}-3 \mathrm{DMpC}$ electrode is even superior to the previous carbon-based anodes (Fig. 3e) $[10,30,40-46]$. To verify the excellent rate performance of the $\mathrm{Se} / \mathrm{N}-3 \mathrm{DMpC}$ anode, $\mathrm{CV}$ measurements with diversity sweep rates between 0.2 and $1.2 \mathrm{mV} \mathrm{s}^{-1}$ were performed. The contribution of surface capacitance gradually ascends from 62.5 to $82.9 \%$ in Se/N-3DMpC electrode with increasing the scan rate, which is greater than those of Se-3DMpC and N-pC at the same sweep rate (Figs. 3f-g and S21-S23). Such high capacitive energy storage behavior of the $\mathrm{Se} / \mathrm{N}$ 3DMpC electrode is highly correlated with the characteristics of its hierarchical porous structure, which is consistent with previous work [47, 48].
Notably, the Se/N-3DMpC electrode maintains a high reversible capacity of $214 \mathrm{mAh} \mathrm{g}^{-1}$ at a high current density of $2 \mathrm{~A} \mathrm{~g} \mathrm{~g}^{-1}$ even after 5,000 cycles, indicating that the $\mathrm{Se} / \mathrm{N}-3 \mathrm{DMpC}$ electrode has excellent durability (Fig. $3 \mathrm{~h}$ ). On the contrary, the performance of the Se-3DMpC and $\mathrm{N}-\mathrm{pC}$ electrodes gradually decayed and only maintained a reversible capacity of 97 and $3 \mathrm{mAh} \mathrm{g}^{-1}$ after 5,000 cycles, which further demonstrated the excellent cycle stability of the Se/N-3DMpC electrode (Fig. S24). The cycle stability of $\mathrm{Se} / \mathrm{N}-3 \mathrm{DMpC}$ is better than most materials reported in the previous literature (Table S2). More importantly, the morphology of the $\mathrm{Se} / \mathrm{N}-3 \mathrm{DMpC}$ electrode did not change significantly after long-term operation, indicating the superior stable structure (Fig. S25). Additionally, electrochemical impedance spectroscopy (EIS) was conducted to further analyze the electrochemical behaviors (Fig. S26). The results show that $\mathrm{Se} / \mathrm{N}-3 \mathrm{DMpC}$ possesses the smallest diameter of semicircle than the others, indicating that charge-transfer resistance $\left(R_{\mathrm{ct}}\right)$ of Se/N-3DMpC is lower than that of Se3DMpC and N-pC. Simultaneously, the slope of straight line of $\mathrm{Se} / \mathrm{N}-3 \mathrm{DMpC}$ electrode is the smallest among all electrodes. The results prove the additional Se/ $\mathrm{N}$ dopants in the carbon-based materials can contribute to fast $\mathrm{K}^{+}$transport kinetics.

\subsection{Reversibility and Kinetic Analysis of Se/N-3DMpC Anode}

Figure 4a-c displays the ex situ Raman spectra during the second discharging/charging cycle and the corresponding $\mathrm{I}_{\mathrm{D}} /$ $\mathrm{I}_{\mathrm{G}}$ ratio. The intensity of $\mathrm{D}$ and $\mathrm{G}$ bands gradually weakens during the discharging process owing to the formation of $\mathrm{KC}_{\mathrm{x}}$ which could narrow the depth of optical skin [43]. In the depotassiation process, the peaks intensity could return to the pristine state, suggesting the excellent reversibility of the architecture. At the same time, compared with the pristine one, the $I_{\mathrm{D}} / I_{\mathrm{G}}$ value of Se/N-3DMpC increased after potassiation (Fig. 4c), which derives from the augment of disordered state with the insertion of $\mathrm{K}^{+}$[49]. With the extraction of $\mathrm{K}^{+}$, the value decreases again, indicating that the structure has good reversibility. Additionally, ex situ XRD showed that the (002) peak slightly shifted negatively during the discharging process, and can be returned to the original position during the charging process, revealing 

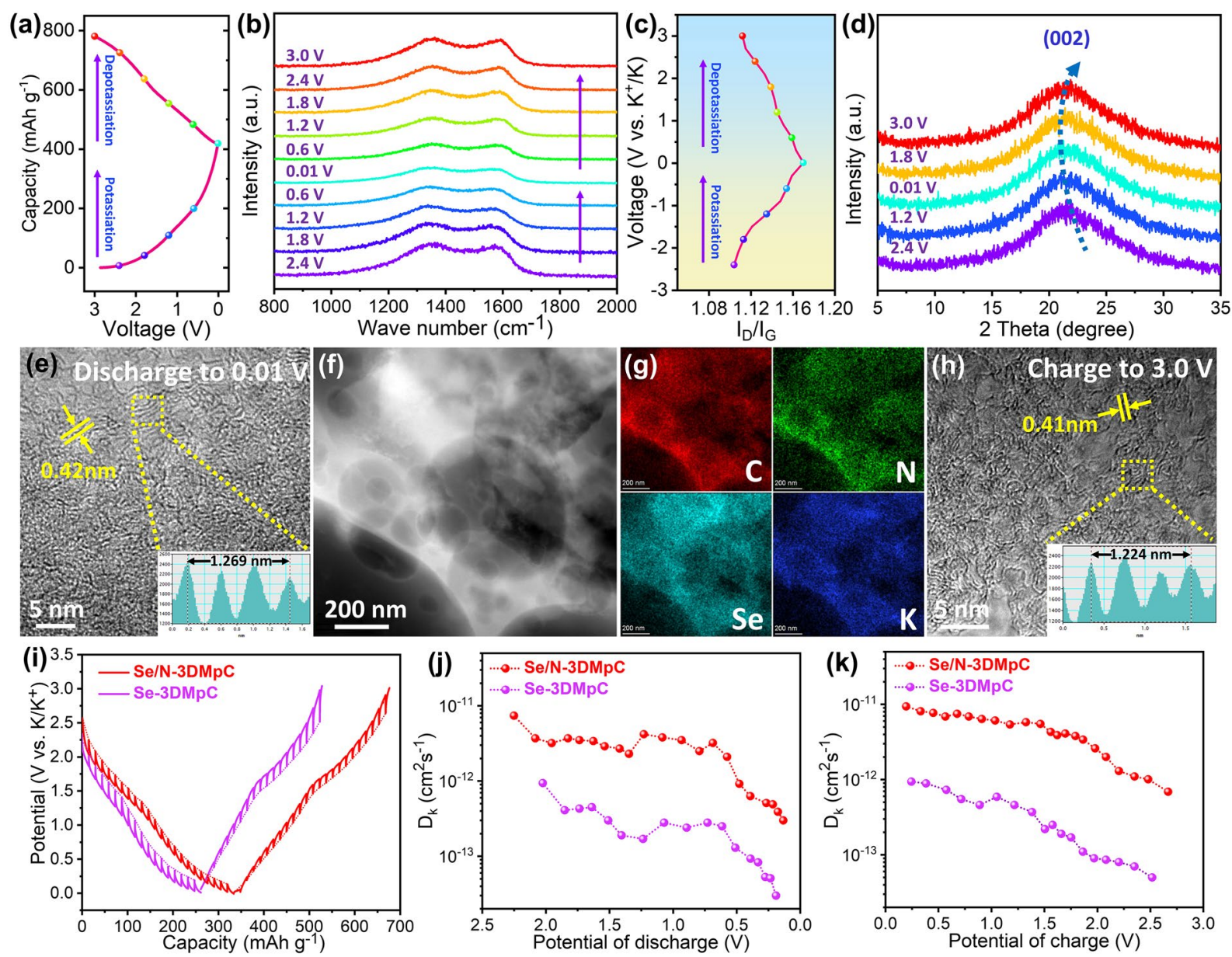

Fig. 4 a Second discharge/charge cycle and the corresponding voltage position, b ex situ Raman spectra at 0.2 A $\mathrm{g}^{-1}$, c corresponding $\mathrm{I}_{\mathrm{D}} / \mathrm{I}_{\mathrm{G}}$ ratio, $\mathbf{d}$ ex situ XRD patterns, e HRTEM at fully discharged, $\mathbf{f}$ HAADF-STEM image, $\mathbf{g}$ elemental mappings, and $\mathbf{h}$ HRTEM at fully charged of $\mathrm{Se} / \mathrm{N}-3 \mathrm{DMpC}$. i Galvanostatic intermittent titration technique (GITT) curves of Se/N-3DMpC and Se-3DMpC at the 200th cycles. The K-ion diffusion coefficient of $\mathbf{j} \mathrm{Se} / \mathrm{N}-3 \mathrm{DMpC}$ and $\mathbf{k} \mathrm{Se}-3 \mathrm{DMpC}$ calculated by the GITT during potassiation and depotassiation processes

that the insertion of $\mathrm{K}^{+}$would only cause the mild expansion of interlayer spacing owing to the enlarged carbon interlayer distance (Fig. 4d) [50]. This is consistent with the phenomenon observed in ex situ (HR)TEM and SEM measurements (Figs. 4e-h and S27). In addition, the morphology of the Se/N-3DMpC electrode did not change significantly (Fig. S27), and the interlayer spacing was slightly enlarged to $0.42 \mathrm{~nm}$ (Fig. 4e). The EDS mappings manifest that $\mathrm{K}, \mathrm{Se}, \mathrm{N}$, and $\mathrm{C}$ distribute uniformly over the electrode materials (Figs. 4f-g and S27), which further confirms potassium ions are uniformly embedded in Se/N-3DMpC electrode. The interlamellar distance of $\mathrm{Se} / \mathrm{N}-3 \mathrm{DMpC}$ returned to $0.41 \mathrm{~nm}$ upon depotassiation (Fig. 4h), implying the expansion of interlayer spacing caused by chemical bonds in $\mathrm{Se} / \mathrm{N}-3 \mathrm{DMpC}$ could withstand repeated insertion and extraction of potassium ions without drastic changes. The $e x$-situ XPS spectra were analyzed to study the related properties of the $\mathrm{Se} / \mathrm{N}-3 \mathrm{DMpC}$ electrode during the electrochemical process. In the first fully discharged state, the peaks of Se $3 d_{5 / 2}$ and $S e 3 d_{3 / 2}$ decrease and shift negatively, which indicates the strong interaction between doped Se and K (Fig. S28a). In the first fully charged state, these peaks show positive displacement, and the peak positions almost remain unchanged after 200 cycles, which verifies the stable structure of Se/N-3DMpC (Fig. S28a). Moreover, the high-resolution spectrum of $\mathrm{N} 1 \mathrm{~s}$ of $\mathrm{Se} / \mathrm{N}-3 \mathrm{DMpC}$ electrode shifted negatively at discharged state and can be returned to the original position at fully charged state, further proving 
the excellent reversibility of the $\mathrm{Se} / \mathrm{N}-3 \mathrm{DMpC}$ electrode (Fig. S28b).

The galvanostatic intermittent titration technique (GITT) was employed to further investigate the potassium-ion diffusion coefficient $\left(\mathrm{D}_{\mathrm{k}}\right)$ in $\mathrm{Se} / \mathrm{N}-3 \mathrm{DMpC}$ and $\mathrm{Se}-3 \mathrm{DMpC}$ during charging/discharging process (Figs. $4 \mathrm{i}$ and S29) [51]. The $D_{\mathrm{k}}$ values in $\mathrm{Se} / \mathrm{N}-3 \mathrm{DMpC}$ electrode are higher than those of Se-3DMpC electrode at all potentials during cycling (Fig. $4 \mathrm{j}, \mathrm{k}$ ). The enhanced diffusion rate of potassium ions can be rationally attributed to the expanded ionic diffusion channels and porous network. To further get deeper atomic insight into the fast kinetics for the Se/N-3DMpC, the diffusion barrier energies $\left(E_{\mathrm{b}}\right)$ of $\mathrm{K}^{+}$in the synthesized samples have been investigated by DFT method (Fig. S30). The diffusion path of potassium ion between the two representative neighboring hollow sites in the pure-C, N-pC, Se-3DMpC, and Se/N-3DMpC is shown in Figs. S31-S34, respectively. It should be noted that the $\mathrm{Se} / \mathrm{N}-3 \mathrm{DMpC}$ delivers the lowest diffusion energy barrier $(0.27 \mathrm{eV})$ compared with that of Se3DMpC $(0.32 \mathrm{eV}), \mathrm{N}-\mathrm{pC}(0.51 \mathrm{eV})$, and pure-C $(0.54 \mathrm{eV})$, confirming the introduction of $\mathrm{Se}$ and $\mathrm{N}$ dopants conduces to decrease the diffusion resistance of $\mathrm{K}^{+}$.

\subsection{Structure and Electrochemical Performance of A-Se/N-3DMpC Cathode}

The Se/N-3DMpC was then activated by $\mathrm{KOH}$ in order to increase the SSA so that it can adsorb more anions. The as-obtained activated Se/N-3DMpC (A-Se/N-3DMpC) was employed as the capacitor-type cathode of the PIHCs. The 3D hierarchical porous morphology of $\mathrm{A}-\mathrm{Se} / \mathrm{N}-3 \mathrm{DMpC}$ is well maintained, and a plenty of pores are evenly dispersed in the hierarchical porous architecture (Fig. S35a-e). The EDS mappings and XPS analysis confirm that the heteroatoms of Se and $\mathrm{N}$ still remain in the $\mathrm{A}-\mathrm{Se} / \mathrm{N}-3 \mathrm{DMpC}$ (Figs. S35f-i and S36a-c), which could furnish abundant active sites for adsorption/desorption of anions and afford additional pseudocapacitive energy storage. Furthermore, the DFT calculation suggests that adsorption energy of anions on the A-Se/N-3DMpC is stronger than that on the pure carbon, indicating that $\mathrm{Se} / \mathrm{N}$ co-doping conduces to the adsorption of anions on the carbon (Fig. S37a, b). Also, charge density difference was conducted to analyze the transferred charges between adsorbed $\mathrm{PF}_{6}{ }^{-}$and the different carbon materials. More transferred charges can be observed between adsorbed $\mathrm{PF}_{6}{ }^{-}$and $\mathrm{Se} / \mathrm{N}$ co-doped carbon, indicating that $\mathrm{Se} / \mathrm{N}$ co-doping is conducive to improving the adsorption ability of anions. The $\mathrm{N}_{2}$ adsorption-desorption report demonstrates that the SSA of $\mathrm{A}-\mathrm{Se} / \mathrm{N}-3 \mathrm{DMpC}$ is as high as $1453.75 \mathrm{~m}^{2} \mathrm{~g}^{-1}$ (Fig. S36d), much higher than that of the Se/N-3DMpC, implying that the A-Se/N-3DMpC can provide a large number of active sites for anion adsorption. The CV profiles of the A-Se/N-3DMpC present a quasirectangular shape at diversity sweep rates (Fig. S38a), well reflecting a capacitive behavior [52]. Additionally, the rate performance of the A-Se/N-3DMpC cathode exceeds that of the AC previously reported, suggesting an excellent electrochemical reversibility (Fig. S38b, c) [37]. Moreover, 82.6\% of the capacity retention with nearly $100 \%$ Coulombic efficiency of A-Se/N-3DMpC is achieved after 4000 cycles at $1.0 \mathrm{~A} \mathrm{~g}^{-1}$ (Fig. S38d), which makes it be an ideal cathode material for high-performance PIHCs.

\subsection{Electrochemical Performance Tests in PIHCs}

A full-cell PIHC was assembled with A-Se/N-3DMpC as the capacitive-type cathode and $\mathrm{Se} / \mathrm{N}-3 \mathrm{DMpC}$ as the battery-type anode (Figs. 5a and S39). Figure 5a shows the schematic diagram of the A-Se/N-3DMpC//Se/N-3DMpC $\mathrm{PIHC}$, where $\mathrm{K}^{+}$is inserted/extracted into/from the $\mathrm{Se} / \mathrm{N}$ $3 \mathrm{DMpC}$ anode, while $\mathrm{PF}_{6}{ }^{-}$is adsorbed/desorbed on the surface of A-Se/N-3DMpC cathode during charging/discharging. In order to obtain the stable electrochemical performance for PIHCs, the operating voltage was optimized to be in the window range of 0.01-4.0 V (Fig. S40). The PIHCs exhibited a near-rectangular shape CV curves (Fig. 5b) and quasi-triangular shape GCD originates from the coupling effect of Faradaic and non-Faradaic processes (Fig. 5c) [53, 54]. Moreover, the A-Se/N-3DMpC//Se/N3DMpC PIHCs displayed the highest energy density of 186 $\mathrm{Wh} \mathrm{kg}^{-1}$ (at power density of $100 \mathrm{~W} \mathrm{~kg}^{-1}$ ) and can maintain an energy density of $91 \mathrm{Wh} \mathrm{kg}^{-1}$ even at a high power density of $8100 \mathrm{~W} \mathrm{~kg}^{-1}$, which surpass most of the previously reported PIHCs (Fig. 5d) [17, 20, 24, 34, 53, 55-58]. The excellent energy and power densities output of the PIHCs can bridge the gap between high-energy rechargeable batteries and high-power supercapacitors (Fig. S41). Moreover, the optimized A-Se/N-3DMpC//Se/N-3DMpC PIHCs could retain $83.2 \%$ of capacity after 5000 cycles at a current density of $1 \mathrm{~A} \mathrm{~g}^{-1}$ (Fig. 5e). Meanwhile, the 
(a)

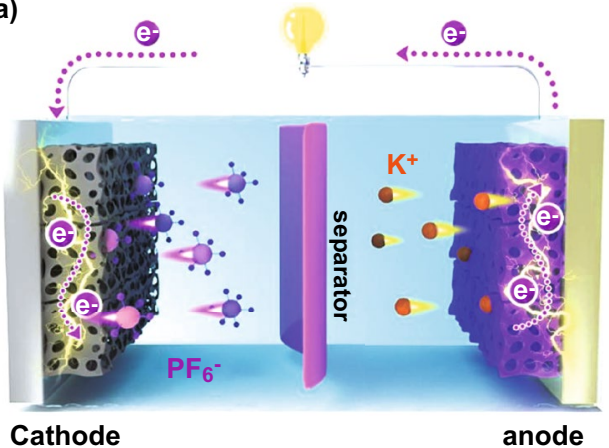

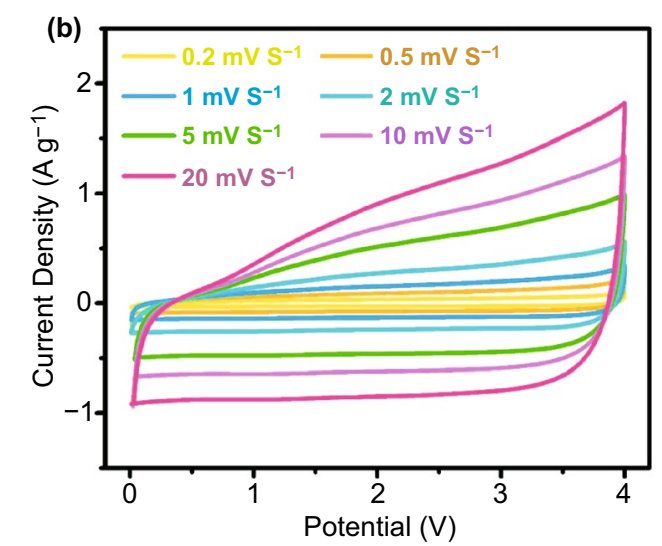

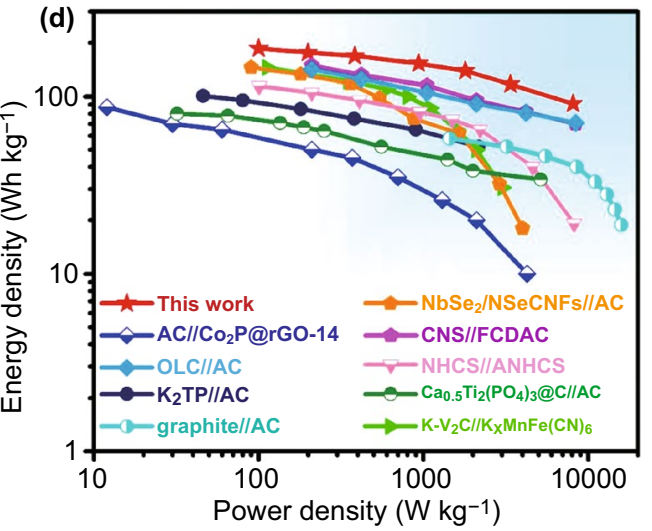

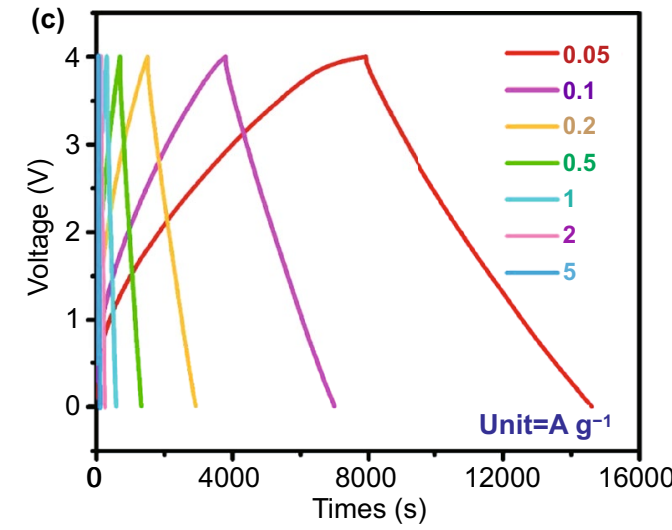

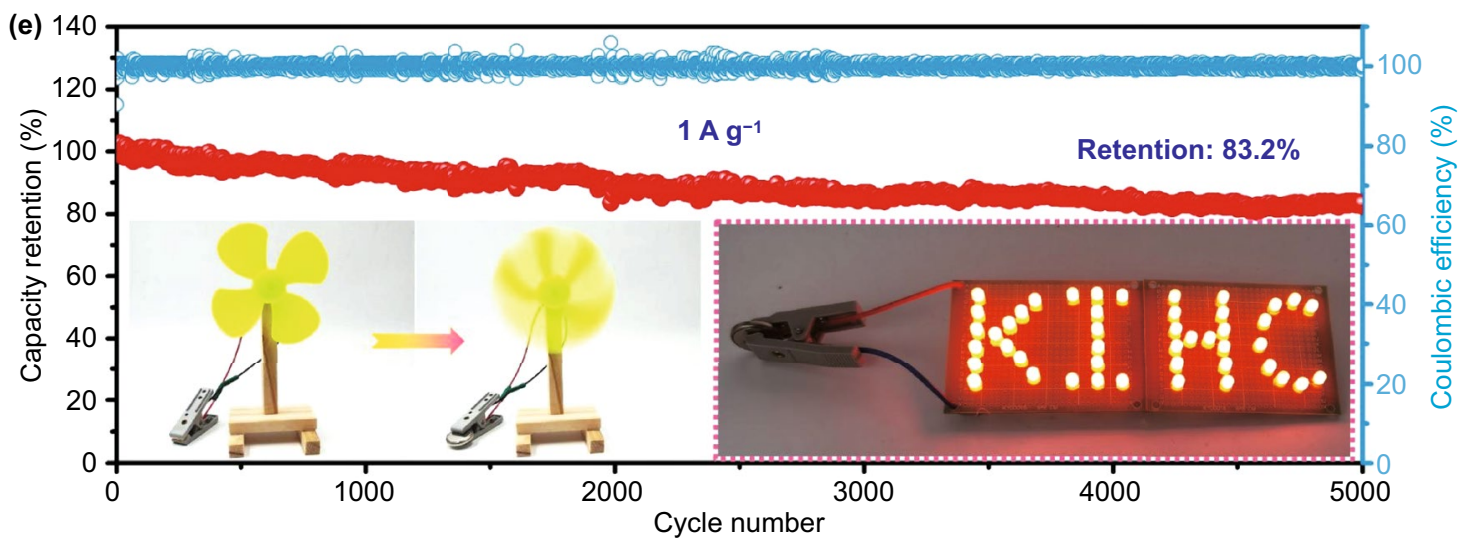

Fig. 5 a Schematic illustration of the A-Se/N-3DMpC//Se/N-3DMpC PIHCs device. b CV curves at various sweep rates and $\mathbf{c}$ charge/discharge curves at diversity current density of the A-Se/N-3DMpC//Se/N-3DMpC PIHCs. d Comparison of the energy/power density of the A-Se/N$3 \mathrm{DMpC} / / \mathrm{Se} / \mathrm{N}-3 \mathrm{DMpC} \mathrm{PIHCs}$ with the reported works. e Long-term cycle performance of the PIHCs at $1 \mathrm{~A} \mathrm{~g}^{-1}$. Inset: Photograph of miniwindmill and LED powered by the A-Se/N-3DMpC//Se/N-3DMpC PIHCs

cycling stability of the A-Se/N-3DMpC//Se/N-3DMpC PIHCs exceeds most of the previously reported PIHCs (Table S3). Notably, this A-Se/N-3DMpC//Se/N-3DMpC PIHCs device can easily actuate a mini-windmill and light up a "KIHC" logo including 46 light-emitting diodes (LEDs) in parallel (in inset of Fig. 5e), further demonstrating the potential practical application of the PIHCs.
According to the above analysis, the outstanding electrochemical properties of PIHCs using Se/N-3DMpC and A-Se/N-3DMpC electrodes can be legitimately ascribed to their distinctive structure and component advantages: (1) 3D hierarchical porous architecture can afford adequate contact area between the electrolyte and the active sites to facilitate the transport of electrolyte and ions; (2) 3D 
framework carbon potentially provides more capability by an electrical double-layer capacitance, which could reinforce the $\mathrm{K}^{+}$storage capacity for PIHCs; (3) the enlarged interlayer space is conducive to the intercalation/extraction of large-size K ions; and (4) the numerous defects and additional active sites caused by the co-doping of Se and $\mathrm{N}$ can be beneficial to improve the adsorption capability of cations/anions, resulting in the rapidly electrochemical reaction kinetics for anode and improved capacity for cathode.

\section{Conclusions}

In summary, a reliable and versatile self-sacrificial template method was developed to fabricate $\mathrm{Se}$ and $\mathrm{N}$ co-doped 3D macroporous carbon (Se/N-3DMpC). Benefiting from the advantages of 3D hierarchical porous architecture, enlarged interlayer space and abundant heteroatoms doping, Se/N3DMpC exhibited a high reversible capacity and attractive kinetic property for potassium-ion storage. Meanwhile, the profusion active sites and hierarchical porous structure as well as the ultrahigh surface area of $\mathrm{A}-\mathrm{Se} / \mathrm{N}-3 \mathrm{DMpC}$ endow it with high specific capacitance as cathode of PIHCs. Taking advantages of the Se/N-3DMpC anode and the A-Se/N3DMpC cathode, we demonstrate a high-performance PIHC that was capable of delivering high energy density of $91 \mathrm{Wh}$ $\mathrm{kg}^{-1}$ at a high power density of $8100 \mathrm{~W} \mathrm{~kg}^{-1}$ and exhibit outstanding cycling stability. This work may open a new avenue for fabricate advanced materials available for a variety of applications.

Acknowledgements This work was supported by the National Natural Science Foundation of China (Project No. 21875253), CASCommonwealth Scientific and Industrial Research Organization (CSIRO) Joint Research Projects (121835KYSB20200039), and Scientific Research and Equipment Development Project of CAS (YJKYYQ20190007).

Open Access This article is licensed under a Creative Commons Attribution 4.0 International License, which permits use, sharing, adaptation, distribution and reproduction in any medium or format, as long as you give appropriate credit to the original author(s) and the source, provide a link to the Creative Commons licence, and indicate if changes were made. The images or other third party material in this article are included in the article's Creative Commons licence, unless indicated otherwise in a credit line to the material. If material is not included in the article's Creative Commons licence and your intended use is not permitted by statutory regulation or exceeds the permitted use, you will need to obtain permission directly from the copyright holder. To view a copy of this licence, visit http://creativecommons.org/licenses/by/4.0/.

Supplementary Information The online version contains supplementary material available at https://doi.org/10.1007/ s40820-021-00659-7.

\section{References}

1. L. Fan, K. Lin, J. Wang, R. Ma, B. Lu, A nonaqueous potassium-based battery-supercapacitor hybrid device. Adv. Mater. 30(20), e1800804 (2018). https://doi.org/10.1002/adma.20180 0804

2. L. Xia, B. Tang, J. Wei, Z. Zhou, Recent advances in alkali metal-ion hybrid supercapacitors. Batter. Supercaps (2021). https://doi.org/10.1002/batt.202000332

3. C. Chen, Z. Wang, B. Zhang, L. Miao, J. Cai, Nitrogen-rich hard carbon as a highly durable anode for high-power potassium-ion batteries. Energy Storage Mater. 8, 161-168 (2017). https://doi.org/10.1016/j.ensm.2017.05.010

4. J. Chen, B. Yang, B. Liu, J. Lang, X. Yan, Recent advances in anode materials for sodium- and potassium-ion hybrid capacitors. Curr. Opin. Electrochem. 18, 1-8 (2019). https://doi.org/ 10.1016/j.coelec.2019.07.003

5. J. Ge, B. Wang, J. Wang, Q. Zhang, B. Lu, Nature of $\mathrm{FeSe}_{2} / \mathrm{N}-\mathrm{C}$ anode for high performance potassium ion hybrid capacitor. Adv. Energy Mater. 10(4), 1903277 (2019). https:// doi.org/10.1002/aenm.201903277

6. H. Li, J. Lang, S. Lei, J. Chen, K. Wang et al., A high-performance sodium-ion hybrid capacitor constructed by metalorganic framework-derived anode and cathode materials. Adv. Funct. Mater. 28(30), 1800757 (2018). https://doi.org/10.1002/ adfm.201800757

7. H.-J. Liang, B.-H. Hou, W.-H. Li, Q.-L. Ning, X. Yang et al., Staging Na/K-ion de-/intercalation of graphite retrieved from spent Li-ion batteries: In operando x-ray diffraction studies and an advanced anode material for $\mathrm{Na} / \mathrm{K}$-ion batteries. Energy Environ. Sci. 12(12), 3575-3584 (2019). https://doi.org/10. 1039/c9ee02759a

8. R. Xu, Y. Yao, H. Wang, Y. Yuan, J. Wang et al., Unraveling the nature of excellent potassium storage in small-molecule Se@peapod-like N-doped carbon nanofibers. Adv. Mater. 32(52), e2003879 (2020). https://doi.org/10.1002/adma.20200 3879

9. X. Hu, Y. Liu, J. Chen, L. Yi, H. Zhan et al., Fast redox kinetics in Bi-heteroatom doped 3D porous carbon nanosheets for high-performance hybrid potassium-ion battery capacitors. Adv. Energy Mater. 9(42), 1901533 (2019). https://doi.org/ 10.1002/aenm.201901533

10. Y. Cui, W. Liu, X. Wang, J. Li, Y. Zhang et al., Bioinspired mineralization under freezing conditions: an approach to fabricate porous carbons with complicated architecture and superior $\mathrm{K}(+)$ storage performance. ACS Nano 13(10), 1158211592 (2019). https://doi.org/10.1021/acsnano.9b05284 
11. Z. Xu, M. Wu, Z. Chen, C. Chen, J. Yang et al., Direct structure-performance comparison of all-carbon potassium and sodium ion capacitors. Adv. Sci. 6(12), 1802272 (2019). https://doi.org/10.1002/advs.201802272

12. B. Yang, J. Chen, L. Liu, P. Ma, B. Liu et al., 3D Nitrogendoped framework carbon for high-performance potassium ion hybrid capacitor. Energy Storage Mater. 23, 522-529 (2019). https://doi.org/10.1016/j.ensm.2019.04.008

13. Y. An, Y. Tian, L. Ci, S. Xiong, J. Feng et al., Micron-sized nanoporous antimony with tunable porosity for high-performance potassium-ion batteries. ACS Nano 12(12), 1293212940 (2018). https://doi.org/10.1021/acsnano.8b08740

14. J. Ma, J. Gu, B. Li, S. Yang, Facile fabrication of 2D stanene nanosheets via a dealloying strategy for potassium storage. Chem. Commun. 55(27), 3983-3986 (2019). https://doi.org/ $10.1039 / \mathrm{c} 9 \mathrm{cc} 00332 \mathrm{k}$

15. S. Chong, Y. Wu, C. Liu, Y. Chen, S. Guo et al., Cryptomelane-type $\mathrm{MnO}_{2}$ /carbon nanotube hybrids as bifunctional electrode material for high capacity potassium-ion full batteries. Nano Energy 54, 106-115 (2018). https://doi.org/10. 1016/j.nanoen.2018.09.072

16. M. Yu, H. Shao, G. Wang, F. Yang, C. Liang et al., Interlayer gap widened alpha-phase molybdenum trioxide as high-rate anodes for dual-ion-intercalation energy storage devices. Nat. Commun. 11(1), 1348 (2020). https://doi.org/10.1038/ s41467-020-15216-w

17. F. Ming, H. Liang, W. Zhang, J. Ming, Y. Lei et al., Porous mxenes enable high performance potassium ion capacitors. Nano Energy 62, 853-860 (2019). https://doi.org/10.1016/j. nanoen.2019.06.013

18. N. Sun, Q. Zhu, B. Anasori, P. Zhang, H. Liu et al., Mxenebonded flexible hard carbon film as anode for stable $\mathrm{Na} / \mathrm{K}$ ion storage. Adv. Funct. Mater. 29(51), 1906282 (2019). https:// doi.org/10.1002/adfm.201906282

19. B. Yang, J. Chen, S. Lei, R. Guo, H. Li et al., Spontaneous growth of 3D framework carbon from sodium citrate for high energy- and power-density and long-life sodium-ion hybrid capacitors. Adv. Energy Mater. 8(10), 1702409 (2018). https:// doi.org/10.1002/aenm.201702409

20. J. Chen, B. Yang, H. Li, P. Ma, J. Lang et al., Candle soot: Onion-like carbon, an advanced anode material for a potassium-ion hybrid capacitor. J. Mater. Chem. A 7(15), 92479252 (2019). https://doi.org/10.1039/c9ta01653h

21. D. Qi, Y. Liu, Z. Liu, L. Zhang, X. Chen, Design of architectures and materials in in-plane micro-supercapacitors: current status and future challenges. Adv. Mater. 29(5), 1602802 (2017). https://doi.org/10.1002/adma.201602802

22. L.-F. Chen, Y. Lu, L. Yu, X.W. Lou, Designed formation of hollow particle-based nitrogen-doped carbon nanofibers for high-performance supercapacitors. Energy Environ. Sci. 10(8), 1777-1783 (2017). https://doi.org/10.1039/c7ee00488e

23. B. Wang, E.H. Ang, Y. Yang, Y. Zhang, M. Ye et al., Postlithium-ion battery era: recent advances in rechargeable potassium-ion batteries. Chem. Eur. J. (2020). https://doi.org/10. 1002/chem.202001811
24. X. Liu, G.A. Elia, B. Qin, H. Zhang, P. Ruschhaupt et al., High-power Na-ion and K-ion hybrid capacitors exploiting cointercalation in graphite negative electrodes. ACS Energy Lett. 4(11), 2675-2682 (2019). https://doi.org/10.1021/acsen ergylett.9b01675

25. Z. Jian, Z. Xing, C. Bommier, Z. Li, X. Ji, Hard carbon microspheres: potassium-ion anode versus sodium-ion anode. Adv. Energy Mater. 6(3), 1501874 (2016). https:// doi.org/10.1002/aenm.201501874

26. Z. Jian, W. Luo, X. Ji, Carbon electrodes for K-ion batteries. J. Am. Chem. Soc. 137(36), 11566-11569 (2015). https:// doi.org/10.1021/jacs.5b06809

27. Z. Jian, S. Hwang, Z. Li, A.S. Hernandez, X. Wang et al., Hard-soft composite carbon as a long-cycling and highrate anode for potassium-ion batteries. Adv. Funct. Mater. 27(26), 1700324 (2017). https://doi.org/10.1002/adfm. 201700324

28. Z. Xing, Y. Qi, Z. Jian, X. Ji, Polynanocrystalline graphite: A new carbon anode with superior cycling performance for K-ion batteries. ACS Appl. Mater. Interfaces 9(5), 4343-4351 (2017). https://doi.org/10.1021/acsami.6b06767

29. R.A. Adams, J.M. Syu, Y. Zhao, C.T. Lo, A. Varma et al., Binder-free N- and O-rich carbon nanofiber anodes for long cycle life K-ion batteries. ACS Appl. Mater. Interfaces 9(21), 17872-17881 (2017). https://doi.org/10.1021/acsami.7b02476

30. W. Yang, J. Zhou, S. Wang, Z. Wang, F. Lv et al., A threedimensional carbon framework constructed by N/S co-doped graphene nanosheets with expanded interlayer spacing facilitates potassium ion storage. ACS Energy Lett. 5(5), 16531661 (2020). https://doi.org/10.1021/acsenergylett.0c00413

31. P. Giannozzi, S. Baroni, N. Bonini, M. Calandra, R. Car et al., Quantum espresso: a modular and open-source software project for quantum simulations of materials. J. Phys. Condens. Matter 21(39), 395502 (2009). https://doi.org/10.1088/0953$8984 / 21 / 39 / 395502$

32. J. Ye, H. Zhao, W. Song, N. Wang, M. Kang et al., Enhanced electronic conductivity and sodium-ion adsorption in N/S co-doped ordered mesoporous carbon for high-performance sodium-ion battery anode. J. Power Sources 412, 606-614 (2019). https://doi.org/10.1016/j.jpowsour.2018.12.002

33. B. Huang, L. Xiao, J. Lu, L. Zhuang, Spatially resolved quantification of the surface reactivity of solid catalysts. Angew. Chem. Int. Ed. 55(21), 6239-6243 (2016). https://doi.org/10. 1002/anie.201601824

34. J. Chen, B. Yang, H. Hou, H. Li, L. Liu et al., Disordered, large interlayer spacing, and oxygen-rich carbon nanosheets for potassium ion hybrid capacitor. Adv. Energy Mater. 9(19), 1803894 (2019). https://doi.org/10.1002/aenm.201803894

35. S. Liu, M. Wang, T. Qian, J. Liu, C. Yan, Selenium-doped carbon nanosheets with strong electron cloud delocalization for nondeposition of metal oxides on air cathode of zinc-air battery. ACS Appl. Mater. Interfaces 11(22), 20056-20063 (2019). https://doi.org/10.1021/acsami.9b04870

36. W. Hong, Y. Zhang, L. Yang, Y. Tian, P. Ge et al., Carbon quantum dot micelles tailored hollow carbon anode for fast 
potassium and sodium storage. Nano Energy 65, 104038 (2019). https://doi.org/10.1016/j.nanoen.2019.104038

37. X. Hu, G. Zhong, J. Li, Y. Liu, J. Yuan et al., Hierarchical porous carbon nanofibers for compatible anode and cathode of potassium-ion hybrid capacitor. Energy Environ. Sci. 13(8), 2431-2440 (2020). https://doi.org/10.1039/d0ee00477d

38. X. Huang, J. Deng, Y. Qi, D. Liu, Y. Wu et al., A highlyeffective nitrogen-doped porous carbon sponge electrode for advanced K-Se batteries. Inorg. Chem. Front. 7(5), 1182-1189 (2020). https://doi.org/10.1039/c9qi01506j

39. X. Hu, J. Jia, G. Wang, J. Chen, H. Zhan et al., Reliable and general route to inverse opal structured nanohybrids of carbonconfined transition metal sulfides quantum dots for high-performance sodium storage. Adv. Energy Mater. 8(25), 1801452 (2018). https://doi.org/10.1002/aenm.201801452

40. M. Chen, W. Wang, X. Liang, S. Gong, J. Liu et al., Sulfur/ oxygen codoped porous hard carbon microspheres for highperformance potassium-ion batteries. Adv. Energy Mater. 8(19), 1800171 (2018). https://doi.org/10.1002/aenm.20180 0171

41. Y. Liu, H. Dai, L. Wu, W. Zhou, L. He et al., A large scalable and low-cost sulfur/nitrogen dual-doped hard carbon as the negative electrode material for high-performance potassiumion batteries. Adv. Energy Mater. 9(34), 1901379 (2019). https://doi.org/10.1002/aenm.201901379

42. A. Mahmood, S. Li, Z. Ali, H. Tabassum, B. Zhu et al., Ultrafast sodium/potassium-ion intercalation into hierarchically porous thin carbon shells. Adv. Mater. 31(2), e1805430 (2019). https://doi.org/10.1002/adma.201805430

43. Y. Qian, S. Jiang, Y. Li, Z. Yi, J. Zhou et al., In situ revealing the electroactivity of $\mathrm{P}-\mathrm{O}$ and $\mathrm{P}-\mathrm{C}$ bonds in hard carbon for high-capacity and long-life Li/K-ion batteries. Adv. Energy Mater. 9(34), 1901676 (2019). https://doi.org/10.1002/aenm. 201901676

44. Y. Xu, C. Zhang, M. Zhou, Q. Fu, C. Zhao et al., Highly nitrogen doped carbon nanofibers with superior rate capability and cyclability for potassium ion batteries. Nat. Commun. 9(1), 1720 (2018). https://doi.org/10.1038/s41467-018-04190-z

45. W. Yang, J. Zhou, S. Wang, W. Zhang, Z. Wang et al., Freestanding film made by necklace-like $\mathrm{N}$-doped hollow carbon with hierarchical pores for high-performance potassium-ion storage. Energy Environ. Sci. 12(5), 1605-1612 (2019). https://doi.org/10.1039/c9ee00536f

46. X. Zhou, L. Chen, W. Zhang, J. Wang, Z. Liu et al., Threedimensional ordered macroporous metal-organic framework single crystal-derived nitrogen-doped hierarchical porous carbon for high-performance potassium-ion batteries. Nano Lett. 19(8), 4965-4973 (2019). https://doi.org/10.1021/acs.nanolett. $9 \mathrm{~b} 01127$

47. C. Li, X. Zhang, Z. Lv, K. Wang, X. Sun et al., Scalable combustion synthesis of graphene-welded activated carbon for high-performance supercapacitors. Chem. Eng. J. 414, 128781 (2021). https://doi.org/10.1016/j.cej.2021.128781

48. C. Li, X. Zhang, K. Wang, X. Sun, G. Liu et al., Scalable self-propagating high-temperature synthesis of graphene for supercapacitors with superior power density and cyclic stability. Adv. Mater. 29(7), 1604690 (2017). https://doi.org/10. 1002/adma.201604690

49. L. Liu, Y. Chen, Y. Xie, P. Tao, Q. Li et al., Understanding of the ultrastable K-ion storage of carbonaceous anode. Adv. Funct. Mater. 28(29), 1801989 (2018). https://doi.org/10.1002/ adfm.201801989

50. Y. Li, W. Zhong, C. Yang, F. Zheng, Q. Pan et al., N/S codoped carbon microboxes with expanded interlayer distance toward excellent potassium storage. Chem. Eng. J. 358, 1147-1154 (2019). https://doi.org/10.1016/j.cej.2018.10.135

51. D. Li, X. Ren, Q. Ai, Q. Sun, L. Zhu et al., Facile fabrication of nitrogen-doped porous carbon as superior anode material for potassium-ion batteries. Adv. Energy Mater. 8(34), 1802386 (2018). https://doi.org/10.1002/aenm.201802386

52. Y. Li, Y. Yang, P. Zhou, T. Gao, Z. Xu et al., Enhanced cathode and anode compatibility for boosting both energy and power densities of $\mathrm{Na} / \mathrm{K}$-ion hybrid capacitors. Matter 1(4), 893-910 (2019). https://doi.org/10.1016/j.matt.2019.04.003

53. Z. Zhang, M. Li, Y. Gao, Z. Wei, M. Zhang et al., Fast potassium storage in hierarchical $\mathrm{Ca}_{0.5} \mathrm{Ti}_{2}\left(\mathrm{PO}_{4}\right)_{3} @ \mathrm{C}$ microspheres enabling high-performance potassium-ion capacitors. Adv. Funct. Mater. 28(36), 1802684 (2018). https://doi.org/10.1002/ adfm.201802684

54. X. Chen, X.R. Chen, T.Z. Hou, B.Q. Li, X.B. Cheng et al., Lithiophilicity chemistry of heteroatom-doped carbon to guide uniform lithium nucleation in lithium metal anodes. Sci. Adv. 5(2), aau7728 (2019). https://doi.org/10.1126/sciadv.aau7728

55. M. Chen, L. Wang, X. Sheng, T. Wang, J. Zhou et al., An ultrastable nonaqueous potassium-ion hybrid capacitor. Adv. Funct. Mater. 30(40), 2004247 (2020). https://doi.org/10.1002/ adfm.202004247

56. Y. Luo, L. Liu, K. Lei, J. Shi, G. Xu et al., A nonaqueous potassium-ion hybrid capacitor enabled by two-dimensional diffusion pathways of dipotassium terephthalate. Chem. Sci. 10(7), 2048-2052 (2019). https://doi.org/10.1039/c8sc04489a

57. D. Qiu, J. Guan, M. Li, C. Kang, J. Wei et al., Kinetics enhanced nitrogen-doped hierarchical porous hollow carbon spheres boosting advanced potassium-ion hybrid capacitors. Adv. Funct. Mater. 29(32), 1903496 (2019). https://doi.org/ 10.1002/adfm.201903496

58. Y. Wang, Z. Zhang, G. Wang, X. Yang, Y. Sui et al., Ultrafine $\mathrm{Co}_{2} \mathrm{P}$ nanorods wrapped by graphene enable a long cycle life performance for a hybrid potassium-ion capacitor. Nanoscale Horiz. 4(6), 1394-1401 (2019). https://doi.org/10.1039/c9nh0 $0211 \mathrm{a}$ 